\title{
Risk Evaluation and Mitigation Strategies Requirement
}

National Cancer Institute

\section{Source}

National Cancer Institute. Risk Evaluation and Mitigation Strategies Requirement. NCI

Thesaurus. Code C128494.

Data elements that represent the clinical or administrative activities that a REMS program participant may be required to perform. 